\title{
Infeç̧ão e óbitos de profissionais da saúde por COVID-19: revisão sistemática
}

\author{
Infection and death in healthcare workers due to COVID-19: a systematic review
} Infección y muerte de profesionales de la salud por COVID-19: revisión sistemática

\author{
Geisa Sant'Ana ${ }^{1}$ in https://orcid.org/0000-0001-8914-4972 \\ Aline Mizusaki Imoto ${ }^{1}$ i https://orcid.org/0000-0001-8318-4658 \\ Fábio Ferreira Amorim ${ }^{1}$ io https://orcid.org/0000-0003-0929-5733 \\ Monica Taminato ${ }^{2}$ i hitps://orcid.org/0000-0003-4075-2496 \\ Maria Stella Peccin ${ }^{3}$ il https://orcid.org/0000-0003-0329-4588 \\ Levy Aniceto Santana ${ }^{1}$ ib hitps://orcid. org/0000-0001-7743-4892 \\ Leila Bernarda Donato Göttems ${ }^{1}$ io hitps://orcid.org/0000-0002-2675-8085 \\ Erika Barbosa Camargo 4 io hitps://orcid.org/0000-0003-1482-8282
}

Como citar:

Sant'Ana G, Imoto AM, Amorim FF, Taminato $M$, Peccin MS, Santana LA, et al. Infecção e óbitos de profissionais da saúde por

COVID-19: revisão sistemática. Acta Paul Enferm. 2020;33:eAPE20200107.

DOI

http://dx.doi.org/10.37689/actaape/2020A00107

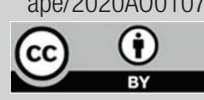

Descritores

Equipamento de proteção individual; Vírus da SARS; Pessoal de saúde; COVID-19

Keywords Personal protective equipment; SARS virus; Health personnel; COVID-19

Descriptors

Equipo de protección personal; Virus del SRAS: Personal de salud ; COVID-19

Submetido

9 de Maio de 2020

Aceito

24 de Junho de 2020

\section{Autor correspondente}

Geisa Sant Ana

E-mail: geisa.s.ana@gmail.com

\section{Resumo}

Objetivo: Identificar as evidências quanto à infecção pelo SARS-CoV-2 e óbitos dos profissionais de saúde e fatores de risco relacionados.

Métodos: Realizou-se uma revisão sistemática buscando-se artigos nas bases de dados Scopus, Portal Regional da BVS, Pubmed e Embase, relacionados à infecção e óbito de profissionais da saúde envolvidos no tratamento de pacientes com a COVID-19. Não houve restrição quanto a data de publicação, idioma ou tipo de estudo. Para a busca foi considerado publicações até 14 de abril de 2020.

Resultados: Foram incluídos 28 artigos. Os fatores de risco mais citados foram: escassez, uso inadequado ou não uso de equipamentos de proteção individual, sobrecarga de trabalho, contato próximo com pacientes e/ou colegas de trabalho potencialmente contaminados, procedimento com risco de geração de aerossol, diagnóstico tardio e renovação de ar ambiente inadequado. De acordo com cada país, o número de profissionais infectados variou de 1.716 a 17.306. Quanto aos óbitos, existem dados de até 605 profissionais falecidos no mundo inteiro. As evidências encontradas são editoriais, estudos transversais e de coorte.

Conclusão: As evidências identificadas demonstram um alto número de profissionais infectados e que foram a óbito, sendo a sobrecarga do sistema de saúde um fator significativo.

\section{Abstract}

Objective: To identify evidences regarding infection by SARS-CoV-2 and deaths of healthcare workers and related-risk factors.

Methods: We conducted a systematic review by searching the following databases SCOPUS, VHL Regional Portal, PubMed and Embase. We included studies on infection and death of healthcare workers who delivery care for COVID-19 patients. There were no restriction on date of publication, idiom, or type of study. The search was conducted until April 14, 2020.

Results: A total of 28 articles were selected. Most mentioned risk factors were: scarcity, inadequate use or non-usage of personal protective equipment, work overload, close contact with potentially infected patients and/or coworkers, risk for aerosol-generating procedures, late diagnosis and inadequate air renovation. According to each country, the number of infected workers ranged from 1,716 to 17,306. Concerning deaths, there are data of up to 605 healthcare workers who died of COVID-19 all over the world. Evidences found were editorials, cross-sectional, and cohort studies. 
Conclusion: Studies identified showed high number of infection and deaths among healthcare workers. Overload of health system was a significant factor.

\section{Resumen}

Objetivo: Identificar evidencias sobre la infección por SARS-CoV-2 y muerte de profesionales de la salud y factores de riesgo relacionados.

Métodos: Se realizó una revisión sistemática en busca de artículos en las bases de datos Scopus, Portal Regional de la BVS, Pubmed y Embase, relacionados con la infección y muerte de profesionales de la salud involucrados en el tratamiento de pacientes con COVID-19. No hubo restricción respecto a la fecha de publicación, idioma ni tipo de estudio. Para la búsqueda se consideraron publicaciones hasta el 14 de abril de 2020.

Resultados: Se incluyeron 28 artículos. Los factores de riesgo más mencionados fueron: escasez, uso inadecuado o falta de uso de equipos de protección individual; sobrecarga de trabajo; contacto cercano con pacientes o compañeros de trabajo potencialmente contaminados; procedimientos con riesgo generador de aerosoles; diagnóstico tardío y renovación inadecuada del aire interior. El número de profesionales infectados varió de 1.716 a 17.306 , de acuerdo con cada país. Respecto a las muertes, hay datos de 605 profesionales fallecidos en todo el mundo. La evidencia se encontró en editoriales, estudios transversales y de cohorte.

Conclusión: Las evidencias identificadas demuestran un alto número de profesionales infectados y fallecidos, y la sobrecarga del sistema de salud es un factor significativo.

\section{Introdução}

A doença pelo novo coronavírus 2019 (COVID-19) com desenvolvimento de síndrome respiratória aguda grave, secundária a infecção pelo SARS-CoV2/2019-nCoV, tem levado a um grave problema de saúde pública e econômico. ${ }^{(1)}$ No final do ano de 2019, a cidade de Wuhan, na província de Hubei, China, apresentou um surto de insuficiência respiratória aguda de pessoas acometidas por um novo coronavírus, que provocou mais de 800 mortes e infectou em torno de 70.000 pessoas nos primeiros 5 dias da epidemia. ${ }^{(2,3)}$ Desde então, ocorreu uma rápida disseminação mundial. A Organização Mundial de Saúde (OMS) declarou Emergência de Saúde Pública de Importância Internacional (ESPII) em 30 de janeiro de 2020 e pandemia em 11 de março de 2020. ${ }^{(4)}$

Em 04 de abril de 2020, o Coronavirus Datacenter do John Hopkins Hospital contabilizava 1.181.825 casos confirmados no mundo, 63.902 mortes e 244.246 casos curados. $^{(4)}$ No Brasil, em 3 de fevereiro de 2020, o Ministério da Saúde (MS) declarou Emergência de Saúde Pública de Importância Nacional (ESPIN) em decorrência da infecção humana pelo SARS-CoV-2, por meio da Portaria MS n ${ }^{\circ} 188$, e conforme Decreto $n^{\circ} 7.616$, de 17 de novembro de 2020. ${ }^{(5,6)}$

A pandemia pela COVID-19 colocou os Profissionais de Saúde (PS) sob extrema pressão com alto potencial de afetar a saúde física e mental.

(7) $\mathrm{O}$ ambiente de trabalho coloca os PS em alto risco para a infecção pelo novo coronavírus, uma vez que a contaminação generalizada de ambientes hospitalares tem sido relacionada a internação de pacientes contaminados pelo SARS-CoV-2, sintomáticos ou não. ${ }^{(8,9)}$ Ong et. al 2020 identificaram que o RNA viral foi detectado em diversas superfícies de um quarto, como interruptores de luz, portas, janelas e vaso sanitário, após a permanência de paciente com COVID-19 sintomático. ${ }^{(8)}$ Outro estudo realizado em ambiente de laboratório mostrou que o SARSCoV-2 sobreviveu mais do que outros vírus respiratórios quando aerossolizado artificialmente. ${ }^{(10)}$

Para que o PS possa desempenhar suas funçóes com segurança, é necessário que sejam disponibilizadas condiçóes organizacionais e ferramentas de trabalho com tecnologias adequadas. Com o propósito de minimizar o risco de infecção cruzada de pacientes e PS, recomenda-se realizar a separação de equipes para cuidar exclusivamente de casos suspeitos e confirmados de COVID-19. ${ }^{(11)} \mathrm{Um}$ estudo realizado em Singapura, que acompanhou o ambiente hospitalar durante a internação de uma criança de 6 meses de idade com exame positivo para SARS-CoV-2 e sem sintomas respiratórios, mantida em isolamento após os pais serem internados por COVID-19, mostrou a contaminação do ambiente mesmo a distância de 1 metro, provavelmente carreada pelos PS que tiveram contato com o cuidado da criança, o que reforça a importância da higiene das mãos. ${ }^{(9)}$

Segundo dados do China's National Health Commission, mais de 3.300 PS foram infectados, sendo que na Itália chegou a 20\%. ${ }^{(12)}$ A citação dos riscos ocupacionais são: exposição ao ambiente 
contaminado, exaustão física e mental causada pela escassez de Equipamento de Proteção Individual (EPI) e perda de pacientes. ${ }^{(7,12,13)}$ Para a redução destes riscos e redução das infecções estão as instruçóes como o uso de EPI e higiene das mãos, o suporte psicológico e a melhoria da logística de suprimentos médicos. ${ }^{(14)}$ Assim, nesse estudo buscou-se sistematizar evidências quanto a infecçóes e óbitos em profissionais de saúde e fatores de risco associados. Partiu-se da pergunta: Quais as evidências acerca das infecçôes, óbitos e fatores de risco dos profissionais de saúde (PS) envolvidos no tratamento de pacientes com a COVID-19?

\section{Métodos}

Trata-se de uma revisão sistemática com protocolo do estudo registrado no site Open Science (https:// osf.io/). O objetivo primário foi identificar os fatores de risco relacionados à infecção e óbitos dos PS durante a pandemia do COVID-19. Os critérios de inclusão estabelecidos foram os estudos relacionados à infecção e óbitos de profissionais de saúde envolvidos no tratamento de pacientes com a COVID-19. Não houve restrição quanto a data de publicação, idioma ou tipo de estudo. Para os dados quanto aos óbitos dos PS, os boletins e documentos técnicos também foram considerados.

\section{Estratégia de busca}

Para identificar a literatura publicada até 14 de abril de 2020, foram aplicadas estratégias de buscas individuais nos seguintes bancos de dados eletrônicos: Portal Regional da BVS, PubMed, SCOPUS, Embase, como descrito no anexo 1. As referências dos artigos incluídos foram rastreadas manualmente para artigos com potencial para inclusão no presente estudo.

\section{Processo de seleção dos estudos}

Os estudos identificados pelas buscas realizadas nas bases de dados previamente citadas foram inseridos no Covidence online software. Duas avaliadoras independentes realizaram a seleção dos estudos com base no título e resumo e após, com base no texto completo. Caso os conflitos não fossem resolvidos entre as duas avaliadoras, uma terceira seria consultada. As referências duplicadas foram identificadas e removidas pelo Covidence online software.

\section{Extração e síntese dos dados}

Os dados extraídos dos artigos foram país e cidade da realização do estudo ou da instituição do primeiro autor, desenho do estudo, dados de infecção, óbitos dos profissionais de saúde e fatores de risco relacionados com a transmissibilidade do vírus. Os dados dos artigos foram extraídos e inseridos em uma tabela no programa Microsoft Excel ${ }^{\circ}$ versão 2016.

\section{Resultados}

Foram identificados 605 estudos dos quais, 173 eram duplicatas. Com base no título e resumo, 432 estudos foram avaliados, 367 excluídos e 65 estudos avaliados na etapa do texto completo. Para essa revisão sistemática rápida, 28 estudos foram incluídos. A razão para todas as exclusóes foi a não resposta do artigo à pergunta da pesquisa. Todos os conflitos foram resolvidos entre as duas pesquisadoras. $\mathrm{O} f l u-$ xograma segundo o PRISMA ${ }^{(15)}$ dos estudos pode ser visualizado conforme apresentado no anexo 2 .

Os 28 estudos incluídos foram publicados no ano de 2020. Os países de realização foram: China $(n=14)$, Estados Unidos $(n=3)$, Itália $(n=3)$, Austrália $(n=1)$, Reino Unido $(n=1)$, Singapura $(\mathrm{n}=3)$, Suécia $(\mathrm{n}=1)$ e Tailândia $(\mathrm{n}=2)$. Quanto ao tipo de estudo: retrospectivo $(n=2)$, relato de caso $(n=1)$, estudo coorte $(n=1)$, editorial, carta ao editor e comentário $(n=16)$, estudo transversal $(n=1)$ recomendaçôes com base em evidências $(n=1)$ e relato de linha do tempo $(n=1)$ e dados epidemiológicos e documentos de governos $(\mathrm{n}=5)$.

\section{Fatores de risco relacionados à infecção pelo SARS-CoV-2}

Os fatores de risco relacionados à infecção pelo SARS-CoV-2 mais citados foram: escassez de Equipamentos de Proteção Individual (EPI) $(n=6)$, sobrecarga de trabalho $(n=2)$, uso inadequado ou não uso de EPI e higiene inadequada das mãos 
$(\mathrm{n}=2)$ contato próximo com pacientes e/ou colegas de trabalho potencialmente contaminados $(n=1)$, procedimento com risco de geração de aerossol - PGA ( $\mathrm{n}=1)$, diagnóstico de COVID-19 tardio $(n=1)$ e renovação de ar insuficiente em ambiente de pressão negativa $(\mathrm{n}=1)$ (Tabela 1$)$.

Tabela 1. Fatores de risco associado à transmissão

\begin{tabular}{lc}
\hline Fator de risco & $\mathrm{n}(\%)$ \\
\hline Escassez de EPI & $6(42,5)$ \\
Infecção cruzada & $1(7,1)$ \\
PGA & $1(7,1)$ \\
Carga horária laboral & $2(14,2)$ \\
Diagnóstico tardio COVID-19 & $1(7,1)$ \\
EPI e Lavagem das mãos inadequadas & $2(14,2)$ \\
Troca de ar - "pressão negativa" & $1(7,1)$ \\
Total & $14(100)$ \\
\hline
\end{tabular}

PGA - Procedimentos geradores de aerossóis; EPI - Equipamento de proteção individual

Quanto ao local de trabalho, Chu et al 2020 relataram que entre os 54 médicos hospitalizados com diagnóstico de COVID-19 de janeiro a fevereiro de 2020, 39 atuavam em unidades clínicas $(72,2 \%)$, 10 em departamento médico tecnológico (18.5\%), 2 em setor de emergência $(3,7 \%)$ e 3 em outros setores diversos não especificados $(5,6 \%) .{ }^{(16)}$ Já, no estudo de Koh 2020, dos 40 casos em profissionais de saúde, 31 atuavam em enfermaria geral (77.5\%), 7 no setor de emergência (17.5\%) e 2 em unidade de terapia intensiva - UTI (5\%) ${ }^{(17)}$ (Quadro 1).

Quadro 1. Sumário dos locais de contaminação no trabalho (unidade de saúde)

\begin{tabular}{|l|c|c|c|}
\hline $\begin{array}{l}\text { Autor } \\
\text { Ano } \\
\text { País }\end{array}$ & \multirow{2}{*}{$\begin{array}{c}\text { Profissão } \\
\text { Chu et al, }\end{array}$} & $\mathbf{n}(\%)$ & Local \\
\hline China & Médicos & $\mathrm{n}=2 / 54(3.7 \%)$ & Setor de emergência \\
\cline { 3 - 4 } & & $\mathrm{n}=39 / 54(72.2 \%)$ & Outras clínicas \\
\cline { 3 - 4 } & & $\mathrm{n}=10 / 54(18.5 \%)$ & Departamento médico tecnológico \\
\cline { 3 - 4 } & & $\mathrm{n}=3 / 54(5.6 \%)$ & Setores diversos não especificado \\
\hline $\begin{array}{l}\text { Koh, 2020 } \\
\text { China }\end{array}$ & \multirow{2}{*}{$\begin{array}{c}\text { Profissionais de } \\
\text { saúde }\end{array}$} & $31(77.5 \%)$ & Enfermaria Geral \\
\cline { 3 - 4 } & & $7(17.5 \%)$ & Setor de emergência \\
\cline { 3 - 4 } & & $2(5 \%)$ & Unidade de Terapia Intensiva \\
\hline
\end{tabular}

$\mathrm{Ng}$ et al 2020 avaliaram o risco de infecção de PS em uso de máscara (N95 ou cirúrgica) durante procedimentos com geração de aerossol. Destes, $85 \%$ estiveram expostos a esses procedimentos estando protegidos com as máscaras e não foram infectados. O estudo sugere que o uso da máscara, higiene das mãos e outros procedimentos padróes protegem o profissional. Máscaras N95 ou equiva- lente são as recomendadas pela maioria dos especialistas em procedimentos com geração de aerossol. ${ }^{(18)}$

Heinzerling et al 2020 avaliou três PS que desenvolveram infecção após exposições desprotegidas ao primeiro caso adquirido na comunidade de COVID-19 nos Estados Unidos. Destaca que essas exposiçôes foram mais longas e provavelmente ocorreram durante o tratamento com dispositivos nebulizadores. ${ }^{(19)}$

\section{Infecções de profissionais de saúde por COVID-19}

Os dados epidemiológicos relativos à infecção de PS variaram de acordo com o contexto, apresentando relação sobretudo com a falta de EPI em momentos de sobrecarga do sistema de saúde. ${ }^{(20)}$

No início do surto da doença na China, os protocolos de proteção eram inconsistentes e a infecção de PS variava entre 3,5\% a 29\% em hospitais de Wuhan. ${ }^{(21)}$ Durante o feriado do ano novo chinês, até 11 de fevereiro de 2020, havia 1.716 casos confirmados em PS, momento no qual as medidas protetoras já estivessem bem estabelecidas, ainda havia escassez de EPI em relação a demanda dos profissionais. ${ }^{(20)}$ Entre os 2.055 PS infectados na China até 20 de fevereiro de 2020, 88\% (1.809) estavam em Hubei. ${ }^{(22)}$ Em 24 de fevereiro de 2020, o National Health Commission of the People's Republic of China já havia reportado 3.387 casos entre PS, o que correspondia a 4,4\% dos 77.262 casos de COVID-19. (23) Após esse período, com as medidas protetoras em quantidade suficiente e treinamento adequado para os PS, não houve relato de infecção entre os 42.600 PS de outras províncias chinesas que foram atuar no atendimento em Wuhan até 31 de março de 2020. (24) Vale ainda salientar que, segundo relato de Tysome e Bhutta 2020, ${ }^{(25)} 14,8 \%$ dos casos em PS foram graves. Já, segundo Chu et al 2020, 43 de 54 PS hospitalizados por COVID-19 a gravidade foi de 79,6\%(16) (Quadro 2).

Em diversos países, a contaminação de PS é agravada, especialmente pela indisponibilidade de EPI frente a sobrecarga dos sistemas de saúde. Na Itália, 17.306 PS haviam contraído a doença de um total de 162.004 casos de COVID-19 registrados até 16 de abril de $2020(10,7 \%) .{ }^{(26)} \mathrm{Na}$ 
Quadro 2. Registro de casos de infecção e óbitos em profissionais de saúde

\begin{tabular}{|l|c|c|c|c|}
\hline Data do registro & País (Cidade) & $\begin{array}{c}\text { Total de Profissionais de saúde infectados } \\
-\mathbf{n}(\%)\end{array}$ & $\begin{array}{c}\text { Total de casos registrados de } \\
\text { infecção na população geral }-\mathbf{n}\end{array}$ & Óbitos - $\mathbf{n}$ \\
\hline $11 / 02 / 2020$ & China (Wuhan) & $1716(3,5$ a 29) & - & 06 \\
\hline $20 / 02 / 2020$ & China (Hubei) & $1809(88)$ & 2055 & 22 \\
\hline $24 / 02 / 2020$ & China & $3387(4,4)$ & 47.262 & 23 \\
\hline $31 / 03 / 2020$ & China & 0 & 11591 & - \\
\hline $31 / 03 / 2020$ & Itália & - & 162.004 & 61 \\
\hline $16 / 04 / 2020$ & Itália & $17.306(10,7)$ & 38.571 & - \\
\hline $31 / 03 / 2020$ & Espanha & $5400(14)$ & 315.531 & - \\
\hline fev a 09/04/20 & EUA & $9282(19)$ & 1265 & 27 \\
\hline $11 / 04 / 2020$ & Austrália & $159(12,6)$ & 4000 & - \\
\hline $15 / 04 / 2020$ & Brasil & $552(13,8)$ & & \\
\hline
\end{tabular}

Espanha, mais de 5.400 PS foram infectados até o final de março de 2020 , quase $14 \%$ do total de infecçóes no país. ${ }^{(27)}$ Essa situaçáo se repete nos Estados Unidos, conforme relato do Centers for Disease Control and Prevention (CDC), entre os 49.370 casos reportados que possuíam dados referentes a pessoa ser ou não PS $(15,6 \%$ total de 315.531 casos de COVID-19 nos EUA no período de 12 de fevereiro a 9 de abril de 2020), 9.282 eram PS (19\%). ${ }^{(28)} \mathrm{Na}$ Austrália em 11 de abril de 2020, momento de registro das condiçóes epidemiológicas com menor sobrecarga do sistema de saúde, houve o relato de 159 casos em PS no estado de Vitória, o que compreende 7 hospitais e 1 clínica radiológica, correspondendo $12,6 \%$ dos 1.265 casos registrados no estado. ${ }^{(29)}$ Em relaçáo ao Brasil, não foram encontrados dados discriminando o total de infecçôes por COVID-19 em PS na busca realizada. Porém, o Conselho Federal de Enfermagem (COFEN) relatou que 4.000 profissionais de enfermagem haviam se afastado do serviço por COVID-19 suspeita ou confirmada (552 já com confirmação diagnóstica) ${ }^{(30)}$ (Quadro 2).

\section{Óbitos}

$\mathrm{Na}$ China, até o dia 11 de fevereiro de 2020, segundo Koh 2020, havia o relato de 6 óbitos entre os 1.716 casos de COVID-19 em PS. ${ }^{(17)}$ Em 20 de fevereiro de 2020, Wang 2020 relatava 22 óbitos entre 2.055 casos confirmados em PS $(1,07 \%){ }^{(21)}$ Já, nos 3.387 casos de PS ocorridos até 24 de fevereiro de 2020, houve mais 1 óbito até o dia 04 de abril de 2020, totalizando 23 óbitos (0,68\%), com uma média de idade de 55,3 $\pm 13,2$ anos, sendo 13 $(56,5 \%)$ abaixo dos 60 anos de idade. ${ }^{(23)}$
Na Itália, foram relatados 24 óbitos de PS entre 4.284 casos de COVID-19 até o dia 22 de março de $2020(0,6 \%),{ }^{(31)}$ sendo relatados outros 37 óbitos até 31 de março de 2020, totalizando 61 óbitos de PS entre os 11.591 óbitos ocorridos no país. ${ }^{(32)}$ Nos EUA, foram relatados 27 óbitos em PS no período de 12 de fevereiro a 9 de abril de 2020. ${ }^{(24,27)}$ No Brasil, embora os dados sejam escassos, o COFEN relatou 30 óbitos de profissionais de enfermagem por COVID-19 até o dia 11 de abril de 2020. ${ }^{(30)}$

No sítio do Medscape que homenageia os PS falecidos em decorrência da pandemia, havia o registro de 605 óbitos até o dia 22 de abril de 2020, sendo 135 nos EUA (22,3\%), 109 na Itália (18,0\%) e 84 no Irã $(13,9 \%) .{ }^{(33)}$ Nesse registro, há referência de 21 óbitos no Brasil (3,5\%) (Anexo 3).

\section{Discussão}

O objetivo desta revisão sistemática foi identificar as evidências a respeito dos fatores de risco envolvidos na infecção e óbito de PS envolvidos no tratamento de pacientes da COVID-19.

As evidências encontradas advêm da opiniáo de especialistas, identificadas em estudos transversais e retrospectivos. Por tratar-se de uma doença causada por um agente infeccioso só identificado há poucos meses, não havia expectativas de estudos com follow-up adequado.

Em relação aos fatores de risco associados à infecção, ressalta-se o fato que para as medidas de proteção do PS, o uso de EPIs é fundamental. Destacase a importância de treinamentos no manuseio adequado desses equipamentos, como, por exemplo, os processos de colocação e retirada dos EPIs e trei- 
namento presencial do uso de EPI. ${ }^{(14)}$ Entretanto, a rotina e os hábitos que propiciam potencializar a contaminação entre os PS também são altamente relevantes. Nesse sentido deve-se proporcionar uma estratégia com o intuito de modificá-las, conscientizando os PS que a transmissão pode acontecer inclusive durante as refeiçôes e reuniōes em grupo. ${ }^{(34)}$

Embora os dados quanto a infecção e óbitos por COVID-19 em PS ainda sejam iniciais, o comportamento da transmissão na China ao longo dos últimos meses sugere que a adoção das medidas de proteção concomitante aos treinamentos e adequaçáo da carga laboral, possuem boa efetividade no controle da transmissão do SARS-CoV-2 para estes PS. Inicialmente, a maior contaminação foi decorrente do desconhecimento do agente etiológico e forma de contágio, seguido da disponibilização insuficiente de EPI para atender a alta demanda. ${ }^{(20,21)}$ Após a regularização da oferta de EPI associado aos treinamentos quanto ao uso adequado, observou-se queda do registo de novos casos de PS infectados. Outros fatores também contribuíram nesta queda, como a redução da carga de trabalho secundária a menor demanda de pacientes como efeito do isolamento social e o reforço com novos PS de outras localidades que foram deslocados para Whuhan. ${ }^{(24)}$ Esse achado coincide com o observado em outros países, especialmente Itália, Espanha e Estados Unidos, que apresentaram sobrecarga do sistema de saúde. ${ }^{(26)}$

Outro aspecto que chama atenção é a observação que mais da metade dos PS que foram a óbito na China apresentarem menos de $60 \operatorname{anos}^{(23)}$ e a quantidade significativa de casos graves em OS, ${ }^{(25,35)} \mathrm{o}$ que sugere que a exposição continuada ao ambiente com maior risco de transmissão e um possível contágio com carga viral mais elevada possa influenciar a evolução da doença.

Dados da Rede Básica da Secretaria Municipal da Saúde da Cidade de Sáo Paulo formada por 64.694 profissionais mostram que em 22 de abril de 2020, 1.666 PS encontravam-se afastados por apresentarem síndrome gripal; 404 com diagnóstico de SARS-CoV-2 confirmado e ocorrência (acumulada) de 6 óbitos decorrentes da COVID-19. ${ }^{(36)}$

Quanto aos locais de contaminação no trabalho (Tabela 1), observa-se uma maior percentagem de profissionais infectados em enfermarias. Uma hipótese seria o fato de que o comportamento dos profissionais nos ambientes de enfermaria apresenta uma menor rigidez em relação a biossegurança quando comparado aos profissionais em outros locais, como a UTI. ${ }^{(37,38)}$

Quanto às estratégias para lidar com a presente situação de pandemia, podemos citar o fornecimento de EPIs, o treinamento adequado e o reforço no hábito do uso de EPI, a proteção dos olhos e adoção de precaução padrão. ${ }^{(1,12,21,25)}$ Além disso, devem ser instituídas medidas para controle da transmissão de modo universal no ambiente hospitalar, mesmo quando esteja em contato apenas com pacientes sem suspeita de COVID-19 ou com outros trabalhadores. ${ }^{(34)}$ Outras estratégias incluem a restrição ao trabalho com adoção do isolamento nos casos de aparecimento de sintomas, a testagem frequente dos PS, o treinamento intensivo para o manejo dos casos graves e moderados, a mobilização dos profissionais para as áreas mais afetadas, a comunicação clara e fácil e protocolos simples e acessíveis. Estudos recomendam a triagem de febre e possíveis sintomas de COVID-19 no início do turno de trabalho dos PS, priorizando-os para a testagem rápida que devem ser sistematicamente realizados. ${ }^{(31)}$

A presente revisão sistemática rápida foi realizada com base em estudos com baixo nível de evidência, o que pode ser visto como limitação da pesquisa. Tal fato explica-se por ser uma doença com relatos recentes, mais precisamente a partir de dezembro de 2019.

\section{Conclusão}

Os dados relativos à infecção e óbitos por COVID-19 em PS referem-se ao período inicial da pandemia. Essa revisão sistemática rápida sugere que os fatores de risco para contágio incluem a escassez de EPI, a sobrecarga de trabalho, a capacitação insuficiente dos PS quanto ao uso adequado das medidas de proteção, assim como a manutenção de comportamentos com menor atenção aos aspectos que envolvam hábitos de biossegurança. Especialmente, o comportamento dos casos na China, Itália, EUA e Espanha sugere que a sobrecarga do sistema de 
saúde é um fator importante para a infecção de profissionais de saúde. As medidas para a redução dos riscos ainda são limitadas, especialmente em relação a poucos estudos terem abordado o tema e a baixa força das evidências disponíveis até o momento.

\section{Referências}

1. Liu M, He P, Liu HG, Wang XJ, Li FJ, Chen S, et al. [Clinical characteristics of 30 medical workers infected with new coronavirus pneumonia]. Zhonghua Jie He He Hu Xi Za Zhi. 2020;43(3):209-14. Chinese.

2. Shereen MA, Khan S, Kazmi A, Bashir N, Siddique R. COVID-19 infection: Origin, transmission, and characteristics of human coronaviruses. J Adv Res. 2020;24:91-8.

3. Doença pelo Coronavírus 2019. Bol Epidemiol Centro Operações Emerg Saúde Pública. 2020;6:1-23. 4. Bialek S, Boundy E, Bowen V, Chow N, Cohn A, Dowling N, et al.; CDC COVID-19 Response Team. Severe outcomes among patients with coronavirus disease 2019 (COVID-19) - United States, February 12-march 16, 2020. MMWR Morb Mortal Wkly Rep. 2020;69(12):343-6.

5. Brasil. Ministério da Saúde. Portaria $N^{\circ} 188$, de 3 de fevereiro de 2020. Diário Oficial da União; 2020. p. 7042.

6. Brasil D n 7.616 de 17 de novembro de 2011. Dispõe sobre a declaração de Emergência em Saúde Pública de Importância Nacional - ESPIN e institui a Força Nacional do Sistema Único de Saúde - FNSUS [Internet]. Diário Oficial da União. 2011, 18 Nov. p. 18. [citado 2020 Jun 12]. Disponível em: http://www.planalto.gov.br/ccivil_03/_ Ato2011-2014/2011/Decreto/D7616.htm

7. Greenberg N, Docherty M, Gnanapragasam S, Wessely S. Managing mental health challenges faced by healthcare workers during covid-19 pandemic. BMJ. 2020;368:m1211.

8. Ong SW, Tan YK, Chia PY, Lee TH, Ng OT, Wong MS, et al. Air, Surface environmental, and personal protective equipment contamination by severe acute respiratory syndrome coronavirus 2 (SARS-CoV-2) from a symptomatic patient. JAMA. 2020;323(16):3-5.

9. Yung CF, Kam KQ, Wong MS, Maiwald M, Tan YK, Tan BH, et al. Environment and personal protective equipment tests for SARS-CoV-2 in the isolation room of an infant with infection. Ann Intern Med. 2020 Apr 1:M20-0942.

10. Doremalen N van, Bushmake T, Morris DH, Holbrook MG, Gamble A, Williamson BN, et al. Aerosol and surface stability of SARS-CoV-2 as compared with SARS-CoV-1. N Engl J Med. 2020;382(16):1564-1567

11. Gan WH, Lim JW, Koh D. Preventing Intra-hospital infection and transmission of coronavirus disease 2019 in health-care workers. Saf Health Work. 2020 Mar 24. doi: 10.1016/j.shaw.2020.03.001.

12. COVID-19: protecting health-care workers. Lancet. 2020;395(10228):922.

13. Maynard A. Economic aspects of addiction policy. Health Promot. 1986;1(1):61-71.

14. Verbeek JH, Rajamaki B, ljaz S, Sauni R, Toomey E, Blackwood B, et al. Personal protective equipment for preventing highly infectious diseases due to exposure to contaminated body fluids in healthcare staff. Cochrane Database Syst Rev. 2020;4:CD011621.

15. Moher D, Liberati A, Tetzlaff J, Altman DG, The PRISMA Group. Preferred reporting items for systematic reviews and meta-analyses: The PRISMA Statement. PLoS Med. 2009;6(7):e1000097.
16. Chu J, Yang N, Wei Y, Yue H, Zhang F, Zhao J, et al. Clinical characteristics of 54 medical staff with COVID-19: A retrospective study in a single center in Wuhan, China. J Med Virol. 2020;92(7):807-13.

17. Koh D. Occupational risks for COVID-19 infection. Occup Med (Lond). 2020;70(1):3-5.

18. Ng K, Poon BH, Kiat Puar TH, Shan Quah JL, Loh WJ, Wong YJ, et al. COVID-19 and the Risk to Health Care Workers: A Case Report. Ann Intern Med. 2020;172(11):766-7.

19. Heinzerling A, Stuckey MJ, Scheuer T, Xu K, Perkins KM, Resseger $\mathrm{H}$, et al. Transmission of COVID-19 to health care personnel during exposures to a hospitalized patient - Solano County, California, February 2020. MMWR Morb Mortal Wkly Rep. 2020;69(15):472-6.

20. Chen C, Zhao B. Makeshift hospitals for COVID-19 patients: where health-care workers and patients need sufficient ventilation for more protection. J Hosp Infect. 2020;105(1):98-9.

21. Wang J, Zhou M, Liu F. Reasons for healthcare workers becoming infected with novel coronavirus disease 2019 (COVID-19) in China. J Hosp Infect. 2020;105(1):100-1.

22. World Health Organization (WHO). Report of the WHO-China Joint Mission on Coronavirus Disease 2019 (COVID-19). Genève: WHO; 2020.

23. Zhan M, Qin $Y$, Xue X, Zhu S. Death from Covid-19 of 23 Health Care Workers in China. N Engl J Med. 2020;382(23):2267-8.

24. Chen W, Huang Y. To Protect healthcare workers better, to save more lives. Anesth Analg. 2020 Mar 30;10.1213/ANE.0000000000004834.

25. Tysome JR, Bhutta MF. COVID-19: protecting our ENT workforce. Clin Otolaryngol. 2020;45(3):311-312

26. Task force COVID-19 del Dipartimento Malattie Infettive e Servizio di Informatica, Istituto Superiore di Sanità. Epidemia COVID-19, Aggiornamento nazionale: 16 aprile 2020. Roma; 2020. [cited 2020 Jun 11]. Available from: https://www.epicentro.iss.it/coronavirus/bollettino/ Bollettino-sorveglianza-integrata-COVID-19_16-aprile-2020.pdf.

27. Stone TE, Kunaviktikul W, Omura M, Petrini M. Editorial: Facemasks and the Covid 19 pandemic: What advice should health professionals be giving the general public about the wearing of facemasks? Nurs Health Sci. 2020 Apr 12;10.1111/nhs.12724.

28. CDC COVID-19 Response Team. February 12-April 9, 2020. MMWR Morb Mortal Wkly Rep. 2020;69(15):477-81.

29. Victoria State Government. Health and Human Services. Coronavirus COVID-19 daily update [Internet]. Melbourne: Victoria State Governmen; 2020. [cited 2020 Jun 11]. Available from: https://www.dhhs.vic.gov. au/coronavirus-covid-19-daily-update

30. Conselho Federal de Enfermagem (COFEN). Brasil tem 30 mortes de profissionais de enfermagem por coronavírus e mais de 4 mil afastados pela doença. Brasília (DF):Conselho Federal de Enfermagem; 2020. p. 1-9.

31. Anelli F, Leoni G, Monaco R, Nume C, Rossi RC, Marinoni G, et al. Italian doctors call for protecting healthcare workers and boosting community surveillance during covid-19 outbreak. BMJ. 2020;368:m1254.

32 Chustecka Z. More than 60 doctores in Italy have died in COVIS-19 Pandemic [Internet]. Medscape. 2020 [cited 2020 Apr 24]. Available from: https://www.medscape.com/viewarticle/https://www.medscape. com/viewarticle/927753927753

33. In Memoriam: Healthcare Workers Who Have Died of COVID-19 [Internet]. Medscape. 2020 [cited 2020 Apr 24]. Available from: https:// www.medscape.com/viewarticle/927976

34. Klompas M, Morris CA, Sinclair J, Pearson M, Shenoy ES. Universal masking in hospitals in the Covid-19 Era. N Engl J Med. 2020;382(21):e63. 
35. Deng CX. The global battle against SARS-CoV-2 and COVID-19. Int J Biol Sci. 2020;16(10):1676-7.

36. São Paulo. Prefeitura do Município de São Paulo. Secretaria Municipal da Saúde da Cidade de São Paulo. COVID-19 Boletim quinzenal DE 30/04/2020. Disponível em: https://www.prefeitura.sp.gov.br/cidade/ secretarias/upload/saude/06052020boletim_covid-19_diariov2.pdf
37. Shingler-Nace A, Birch M, Hernandez A, Bradley K, Slater-Myer L. Minimizing hospital-acquired infections and sustaining change. Nursing. 2019;49(10):64-8.

38. Gallas SR, Fontana RT. Biossegurança e a enfermagem nos cuidados clínicos: contribuições para a saúde do trabalhador. Rev Bras Enferm. 2010;63(5):786-92.

Anexo 1. Bases de dados e estratégias de busca

\begin{tabular}{|c|c|c|}
\hline BASE DE DADOS & ESTRATÉGIA & RESULTADO \\
\hline Scopus & $\begin{array}{l}\text { ( title-abs-key ("health personnel") or title-abs-key (personnel, and health) or title-abs-key ( health and care and providers) or title-abs-key ( } \\
\text { health and care and provider) or title-abs-key (provider, and health and care) or title-abs-key (providers, and health and care) or title-abs-key } \\
\text { ( healthcare and providers) or title-abs-key (healthcare and provider) or title-abs-key (provider, and healthcare) or title-abs-key (providers, } \\
\text { and healthcare ) or title-abs-key ( healthcare and workers) or title-abs-key (healthcare and worker ) and title-abs-key ("covid-19") or title- } \\
\text { abs-key ( } 2019 \text { novel and coronavirus and infection) or title-abs-key ( } 2019 \text {-ncov and infection) or title-abs-key (covid-19 and pandemic) or } \\
\text { title-abs-key (coronavirus and disease-19) or title-abs-key (2019-ncov and disease) or title-abs-key (covid19 } 2019 \text { novel and coronavirus and } \\
\text { disease) or title-abs-key (coronavirus and disease 2019)) }\end{array}$ & 91 \\
\hline $\begin{array}{l}\text { Portal Regional } \\
\text { da BVS }\end{array}$ & $\begin{array}{l}\text { ((tw:(Health Personnel)) OR (tw:(Personnel, Health )) OR (tw:(Health Care Providers)) OR (tw:(Health Care Provider)) OR (tw:(Provider, Health Care)) OR } \\
\text { (tw:(Providers, Health Care )) OR (tw:(Healthcare Providers )) OR (tw:(Healthcare Provider)) OR (tw:(Provider, Healthcare)) OR (tw:(Providers, Healthcare } \\
\text { )) OR (tw:(Healthcare Workers)) OR (tw:(Healthcare Worker))) AND ((tw:(COVID-19 )) OR (tw:(2019 novel coronavirus infection)) OR (tw:(2019 novel } \\
\text { coronavirus infection )) OR (tw:(2019-nCoV infection )) OR (tw:(COVID-19 pandemic)) OR (tw:(coronavirus disease-19)) OR (tw:(2019-nCoV disease)) OR } \\
\text { (tw:(COVID19 } 2019 \text { novel coronavirus disease)) OR (tw:(coronavirus disease 2019))) }\end{array}$ & 204 \\
\hline Pubmed & $\begin{array}{l}\text { ((((“"Health Personnel”[Mesh] OR personnel, health OR health care providers OR health care provider OR providers, health care OR providers, health care } \\
\text { OR healthcare providers OR healthcare provider OR providers, healthcare OR providers, healthcare OR healthcare workers OR healthcare worker)))) AND } \\
\text { (((“COVID-19"[Supplementary Concept] OR } 2019 \text { novel coronavirus infection OR } 2019 \text { novel coronavirus infection OR 2019-ncov infection OR covid-19 } \\
\text { pandemic OR coronavirus disease-19 OR 2019-ncov disease OR covid19 } 2019 \text { novel coronavirus disease OR coronavirus disease 2019))) }\end{array}$ & 139 \\
\hline Embase & 'health care personnel'/exp OR 'health care personnel' AND 'covid 19'/exp OR 'covid 19' & 165 \\
\hline
\end{tabular}

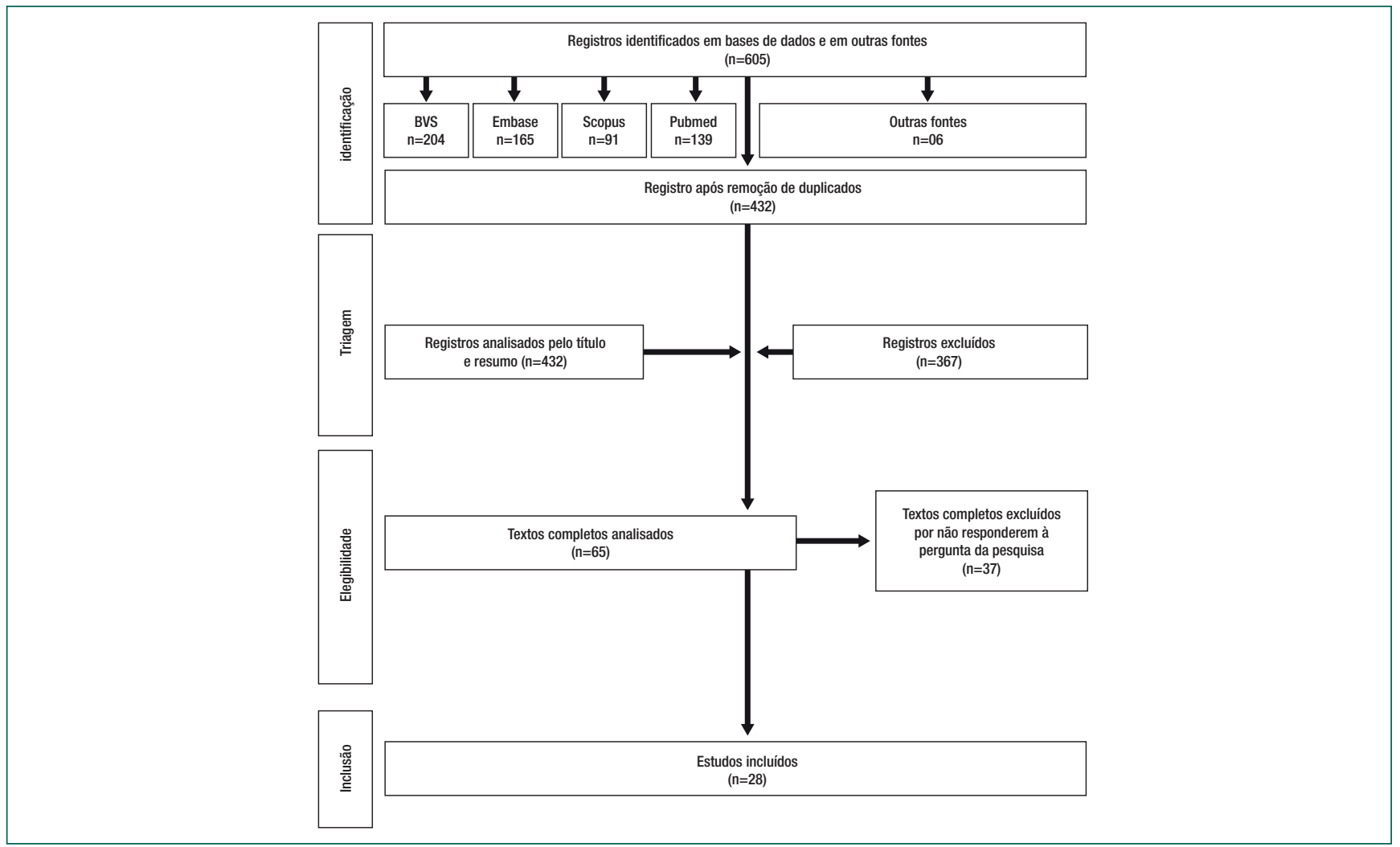

Fonte: Moher D, Liberati A, Tetzlaff J, Altman DG, The PRISMA Group. Preferred reporting items for systematic reviews and meta-analyses: The PRISMA Statement. PLoS Med. 2009;6(7):e1000097. ${ }^{(15)}$

Anexo 2. Fluxograma PRISMA de seleção de estudo e processo de inclusão 


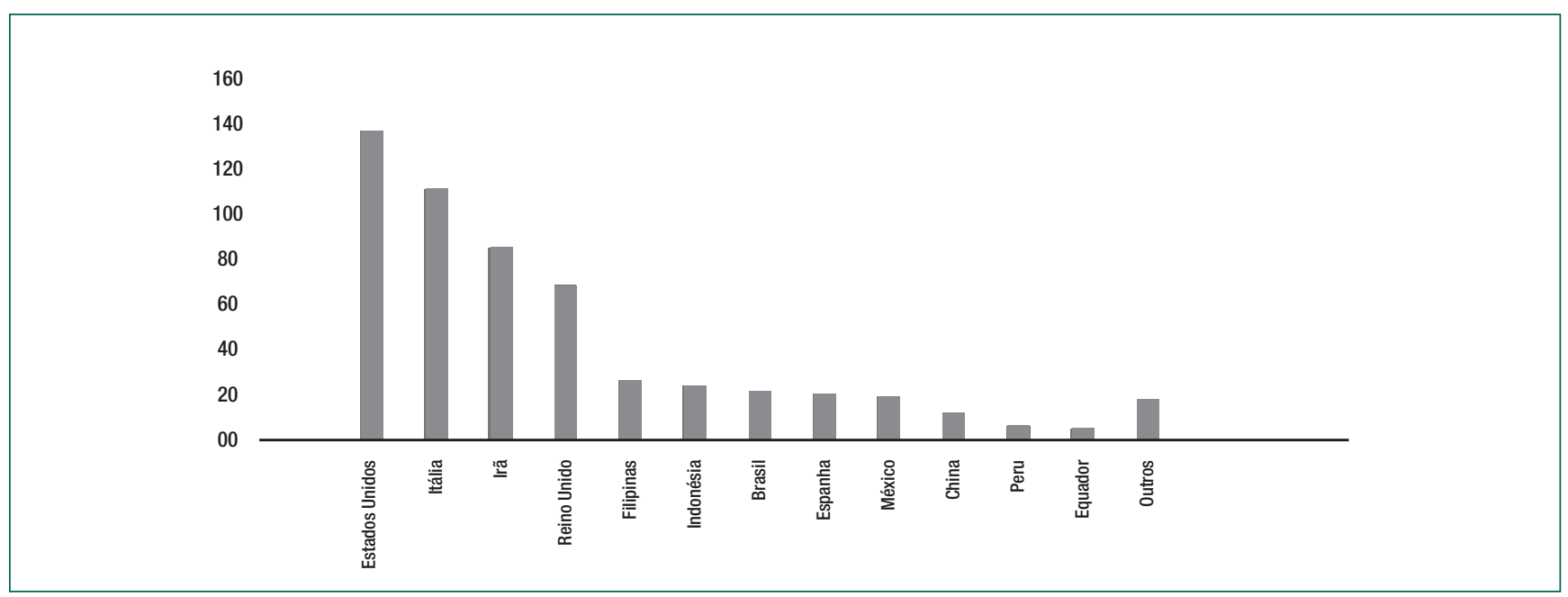

Anexo 3. Óbitos de Profissionais de saúde registrado no Medscape (abr/2020) 